\title{
Design and Construction of an Optical TEM Specimen Holder
}

\author{
Joel Martis, ${ }^{1 *}$ Ze Zhang, ${ }^{1}$ Hao-Kun Li, ${ }^{1}$ Ann Marshall, ${ }^{2}$ Roy Kim, ${ }^{2}$ and Arun Majumdar ${ }^{1,3}$ \\ ${ }^{1}$ Department of Mechanical Engineering, Stanford University, Stanford, CA 94305 \\ ${ }^{2}$ Stanford Nano Shared Facilities, Stanford University, Stanford, CA 94305 \\ ${ }^{3}$ Department of Photon Science, SLAC National Accelerator Laboratory, Menlo Park, CA 94025 \\ *martis@stanford.edu
}

\begin{abstract}
Electron microscopy has enabled atomic resolution imaging of matter. However, unlike optical spectroscopic imaging, traditional electron microscopes provide limited spectroscopic information in terms of their energy resolution. Only recently, owing to advances in monochromated STEM-EELS, have transmission electron microscopes (TEMs) been able to attain a high energy resolution. We recently proposed combining spectrally selective photoexcitation with HRTEM to achieve sub-nanometer scale optical imaging, a technique we called photoabsorption microscopy using electron analysis (PAMELA). To realize PAMELA-TEM experimentally, we constructed a TEM holder with an optical feedthrough, capable of photoexciting materials with different wavelengths. In this article, we describe our process for designing and fabricating an optical TEM specimen holder, highlighting important aspects of the design.
\end{abstract}

Keywords: TEM specimen holder, optical spectroscopy, in situ TEM, optical fiber, photoabsorption microscopy

\section{Introduction}

Optical imaging and spectroscopy provide rich information about the energy states of materials. However, their spatial resolution is limited by the wavelength of (visible) light to about $0.5 \mu \mathrm{m}$. Electron microscopy can achieve subAngstrom resolution but typically provides limited spectral information. Only due to recent developments of electron monochromators, has scanning transmission electron microscopy electron energy loss spectroscopy (STEM-EELS) become a powerful tool for probing energy states of materials [1] . We recently demonstrated $\sim 10 \mathrm{~nm}$ optical imaging in scanning electron microscopy (SEM) via a new imaging technique called photoabsorption microscopy using electron analysis (PAMELA), where spectrally selective photoabsorption modulates secondary electron emission [2]. We also proposed combining spectrally selective photoexcitation with highresolution transmission electron microscopy (HR)TEM (PAMELA-TEM) to achieve sub-nm scale optical imaging [3]. The experimental realization of PAMELA-TEM, however, requires introduction of light inside a TEM with a sufficiently high photon flux to produce a detectable signal.

Light can be introduced in two ways: through a port on the TEM (like the objective aperture) [4,5], or through a TEM specimen holder [6-8]. The former approach requires modification of a TEM column, which can be expensive and potentially detrimental to the instrument, but has the advantage of combining other multifunctional specimen holders (heating, biasing, cryo, etc.) with light input. The latter is advantageous since a TEM holder can be used in any compatible TEM, and the holder can be modified without affecting the TEM column itself. Since the basic principle of PAMELA-TEM requires only a light input, we chose the latter approach and constructed a
TEM holder with light input capabilities. In the following, we explain our approach to designing such a holder, bearing in mind our experimental requirements, vacuum considerations, $\mathrm{X}$-ray safety, and ease of operation.

Several researchers have designed and fabricated TEM holders with light input capabilities in the past [6-8]. These designs have used both free-space optics and fiber-opticsbased approaches. Free-space optics designs offer the possibility of high optical fluxes but require the light source to be rigidly connected to the specimen holder, thereby limiting the light sources that can be used. Fiber-based designs offer the flexibility of using a variety of light sources but are typically limited in the largest optical flux they can achieve owing to their damage threshold. In our case, we chose the fiber-based approach because it met our optical flux requirements and offered other advantages as outlined above.

\section{Methods and Materials}

Prior to designing and fabrication, we outlined the target specifications of our optical TEM specimen holder. These specifications determined the design approach and materials used in the construction of the holder. We designed a holder to fit into a Thermo Fisher Scientific (Portland, OR) TEM based on the following requirements:

- Optical flux of $\sim 10^{7} \mathrm{~W} / \mathrm{m}^{2}$

- Laser spot size $\sim$ between $25-100 \mu \mathrm{m}$

- Laser wavelengths $\sim$ visible light $(400-700 \mathrm{~nm})$

- Motion control $\sim 2 \mathrm{~mm}$ range of motion

- The optical fiber should be easily replaceable

In addition, any TEM holder needs to be vacuumcompatible, X-ray safe, and ideally not affect the resolution and performance of the microscope. Our process flowchart is shown in Figure 1. In the following sections, we discuss each of these design aspects in detail.

Optics. Optical fibers typically have an upper optical flux limit of about $10^{10} \mathrm{~W} / \mathrm{m}^{2}$, which is much higher than our requirement of $10^{7} \mathrm{~W} / \mathrm{m}^{2}$. However, even to achieve an optical flux of $10^{7} \mathrm{~W} / \mathrm{m}^{2}$ at the sample, a focusing optic needs to be used between the fiber and the sample. Given the space constraints due to the objective pole pieces in the TEM, we decided to use a microlens fused to the optical fiber with a working distance to the sample of about $1 \mathrm{~mm}$. The microlens was fabricated with a reflector built in (Figure 2) to help mount the fiber such that it stayed within the space constraints imposed by the objective lens. The microlens assembly was fabricated by WT\&T Inc., Montreal, Canada. The assembly was then inserted into a metal tube and connectorized and polished on the other 


\section{D MLROMAC}

\section{microPREPTM PRO}

\section{High-Speed Sample Preparation}

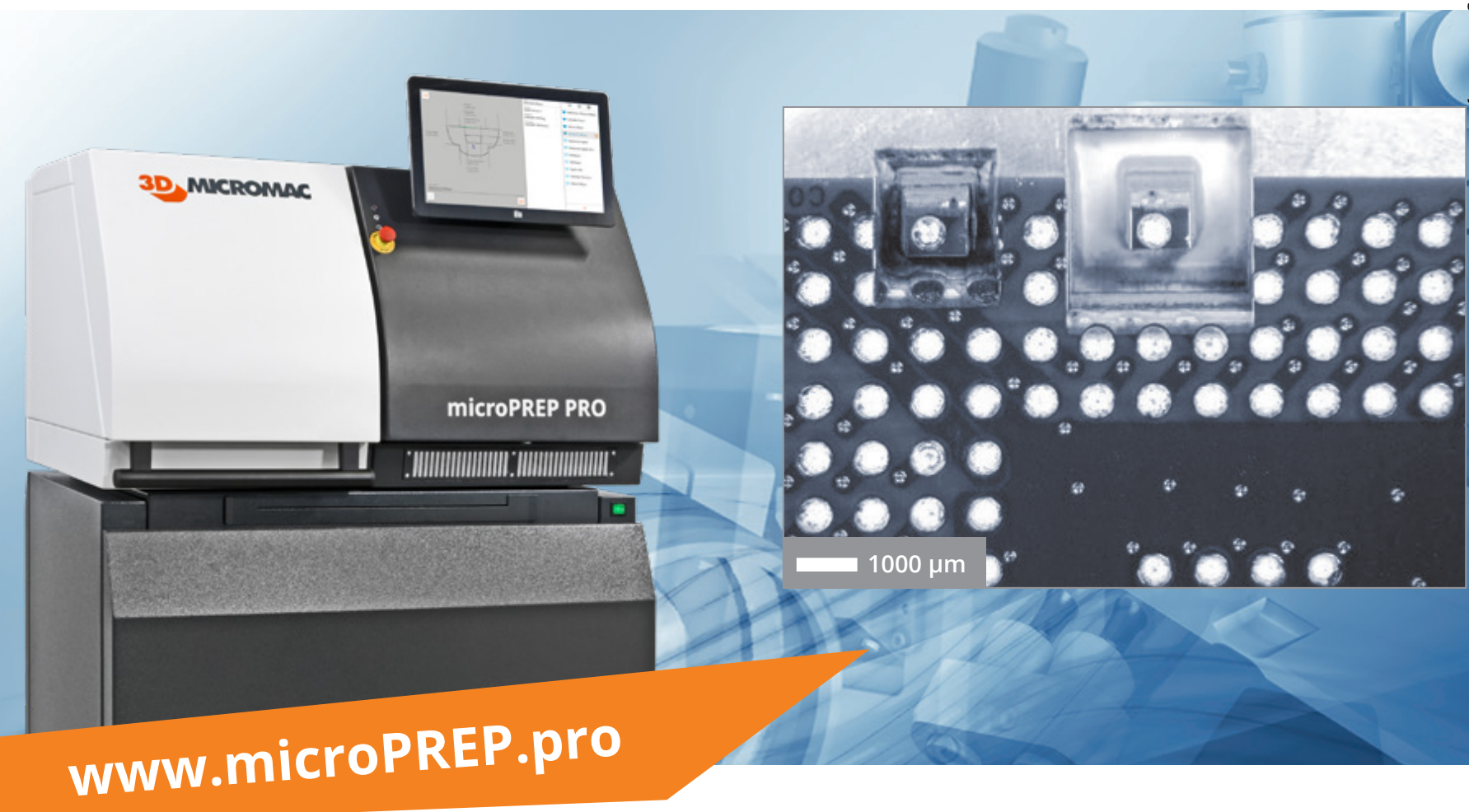

»Free-form laser-based sample preparation

» Larger-sized samples with micron-level precision

»Workflows for TEM, XRM, APT, and micromechanical testing

»Reduces time-to-sample and costs significantly

Contact us now!

Tel: $+4937140043-222$

sales@3d-micromac.com

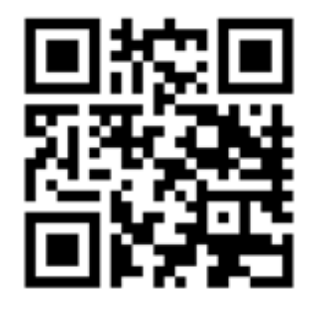


side following the procedure outlined in Thorlabs' Fiber Connectorization Guide [9]. With single-mode fibers, we achieved a spot size of about $20 \mu \mathrm{m}$ at a working distance of $1 \mathrm{~mm}$, and with a $50 \mu \mathrm{m}$ multimode fiber we achieved a spot size of $100 \mu \mathrm{m}$.

Motion control. Since our laser spot size was designed to be between 20 and $100 \mu \mathrm{m}$, which is much smaller than a TEM grid, we needed to be able to control the position of the laser spot on the TEM grid. To achieve this, we developed two designs: (1) the fiber is positioned via a short metal tube held by screws close to the tip of the TEM holder, and (2) the fiber is enclosed in a long metal tube that is attached to a 3-axis external micrometer stage at the rear handle of the holder (Figure 3). The latter has the advantage of letting the user move the fiber in situ, whereas the former is more stable because of the shorter metal tube and is also more affordable. The 3 -axis external micrometer stage was designed in-house using off-the-shelf micrometer screws. In addition to the micrometer screws, we incorporated guide rods to make the stage more stable and reduce drift and backlash.

Vacuum. The specimen chamber of an electron microscope typically operates below a pressure of $10^{-5} \mathrm{mbar}$. The optical feedthrough of the TEM holder needs to have effective vacuum seals, while also allowing for easy switching out of optical fibers. Therefore, we used Viton ${ }^{\circledR}$ o-rings at all temporary vacuum interfaces. For permanent vacuum seals (like the inside of the optical fiber coupler), we used vacuum-compatible epoxies such as TorrSeal $^{\circledR}$ and EPO-TEK ${ }^{\circledR}$ 353ND. These were used in small quantities to minimize any potential contamination on the sample during imaging. For the 3 -axis external micrometer stage, we used metal bellows to allow for motion while maintaining vacuum. The bellows were custom made by Metal Flex Welded Bellows, Inc., Newport, VT.

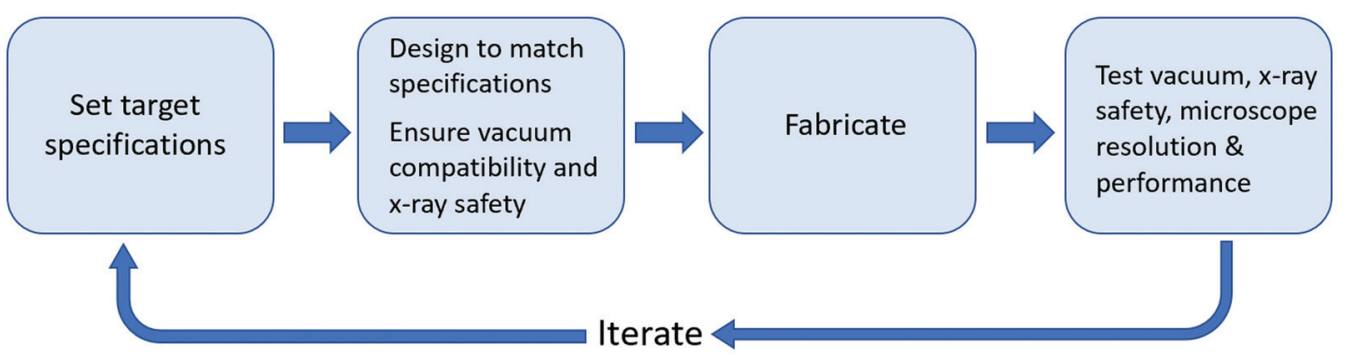

Figure 1: Process flow for constructing a TEM holder.

Schematic Side View:

Ball lens with reflector

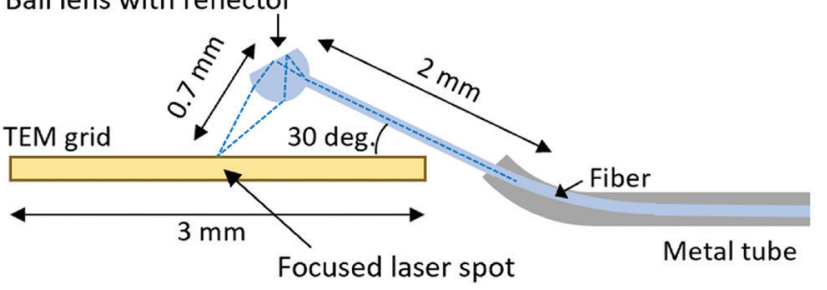

Figure 2: (left) Schematic side view of the optical setup design. (right) Top view of the actual setup.

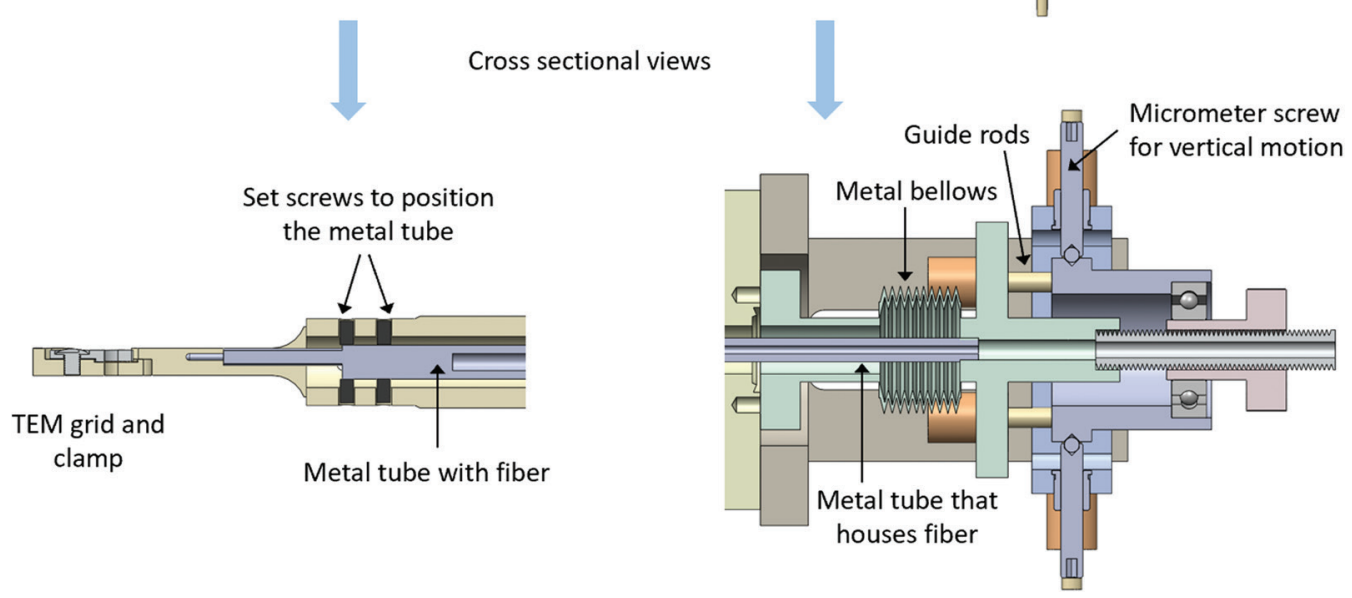

Cross sectional views

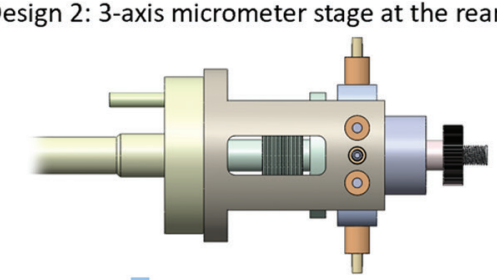

Figure 3: Motion control designs; (left) using fiber positioning screws at the tip of the holder; (right) micrometer stage at the rear handle of the holder.
X-ray safety. TEMs operate between 60 and $300 \mathrm{keV}$, which generates a significant amount of X-rays at the specimen. The TEM holder needs to efficiently shield users from these $\mathrm{X}$-rays. Stainless steel, copper alloys and titanium have X-ray penetration depths of a few $\mathrm{mm}[10]$ at $100 \mathrm{keV}$ and are therefore suitable for shielding. The penetration depth of X-rays through aluminum, however, is a few centimeters, making it unsuitable 
for use. Since certain stainless steels are magnetic and could potentially have interfered with imaging, we chose to fabricate our holder bodies with brass and titanium. The flanges at the rear handle of the holder (purchased off-the-shelf) were made of stainless steel. One important note is that since brass is an alloy of copper and zinc, and zinc outgasses at high temperatures (several hundred degrees Celsius), it is important not to heat a specimen holder made of brass to avoid coating the inside of the specimen chamber with zinc.

Other considerations. Modular design: We chose to implement a modular design, where the holder is made up of a few sections that can be easily assembled and disassembled (Figure 4). The advantage of such a design is that specific parts such as the rear handle or tip can be easily interchanged, and we deemed this important in a research project where our needs and application might change every few months.

TEM grid clamp: Traditional FEI (a company acquired by Thermo Fisher Scientific) single-tilt holders have a springloaded clamp away from the tip of the holder. Since the optical fiber occupies the space right before the grid, we designed a screw-on clamp on the side closer to the tip (Figure 5). Care must be taken to ensure that the screw does not protrude out of the top or bottom of the holder tip.

\section{Results and Discussion}

We fabricated two optical holders-one with a brass body and 3-axis external micrometer stage, and another with a titanium body and fiber positioning screws at the tip (Figure 6). The brass holder was machined at Excel CNC Machining Inc., San Jose, CA, and the titanium holder was machined at the Stanford Thermosciences Machine Shop, Stanford University. Both holders were vacuum-tested using a Hummingbird Scientific (Lacey, WA) high-vacuum leak test station and were found to be leakproof. Following vacuum testing, they were inserted into an FEI Tecnai TEM and tested for X-ray safety by Stanford Environment Health \& Safety and were found to be safe for use.

We also characterized the laser spot size on the sample. Figure 7 shows the laser spot (using a $405 \mathrm{~nm}$ laser) from a singlemode fiber on a piece of filter paper, used in place of a TEM grid. The line profile of the laser intensity shows the spot size to be about $25 \mu \mathrm{m}$. With a laser power of $10 \mathrm{~mW}$, this yields an optical flux of greater than $10^{7} \mathrm{~W} / \mathrm{m}^{2}$, which was our target flux.

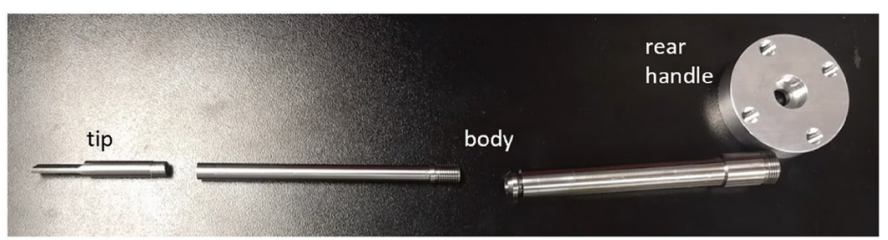

assembly

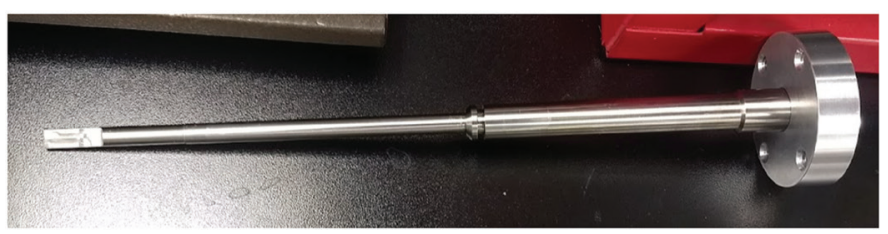

Figure 4: Modular design of the specimen holder; (top) disassembled and (bottom) assembled.

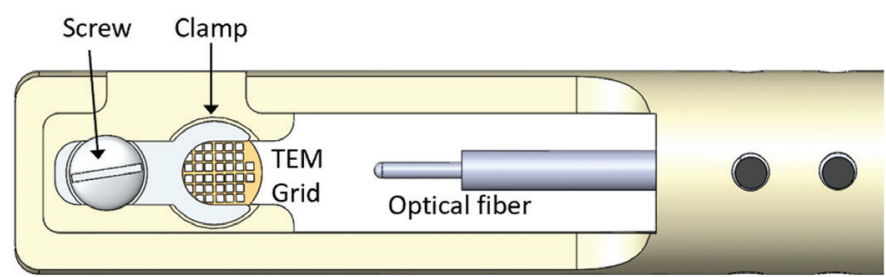

Figure 5: Top view of the tip of the holder showing the design of the TEM grid clamp.

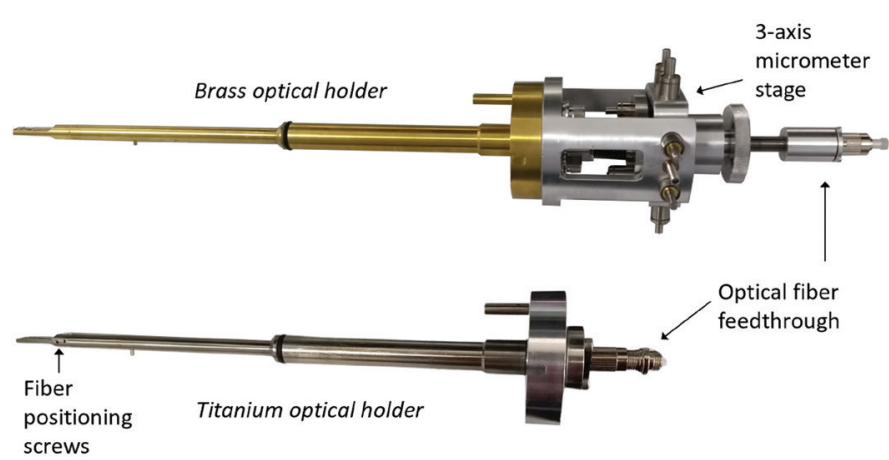

Figure 6: (top) Brass optical holder with a 3-axis micrometer stage at the rear handle and (bottom) titanium optical holder with fiber positioning screws at the tip.

We also tested the TEM holders to see if they impacted the resolution in HRTEM. Figure 8 shows a GaAs cross section (sample courtesy of Chen Shang, Bowers group, UCSB) imaged at $300 \mathrm{keV}$ with its corresponding fast Fourier transform (FFT). In the FFT, diffraction spots are visible beyond $10 \mathrm{~nm}^{-1}$, demonstrating sub-Angstrom lattice fringe resolution.

\section{Conclusion}

In summary, we designed and constructed two TEM specimen holders with optical fiber feedthroughs to enable in situ photoexcitation of materials inside TEMs. We constructed two different motion control mechanisms to enable precise positioning of the laser spot on the TEM grid. Care was taken during materials selection to ensure vacuum compatibility and X-ray safety, and these were tested extensively upon fabrication of the holders. We found that the holders were sufficiently stable in the TEM to enable atomic-resolution imaging.

Our TEM holders are currently supporting proof-ofconcept PAMELA-TEM experiments. In addition to this, we envision them being used to study various kinds of light matter interactions at the atomic scale, such as in situ photocatalysis and light-driven phase transformations. We are now developing TEM holders with electrical bias and light input capabilities, with the additional possibility of cryogenic cooling. We believe that the capability to fabricate TEM specimen holders quickly at an affordable cost will expedite research and enable new possibilities in electron microscopy.

\section{Acknowledgements}

The authors would like to acknowledge and thank Els Kok (Thermo Fisher Scientific, The Netherlands), Kate Marusak (Protochips, Cary, NC), and Brad Takasuka (Silicon Valley Peripherals, San Jose, CA) for helpful discussions. The 

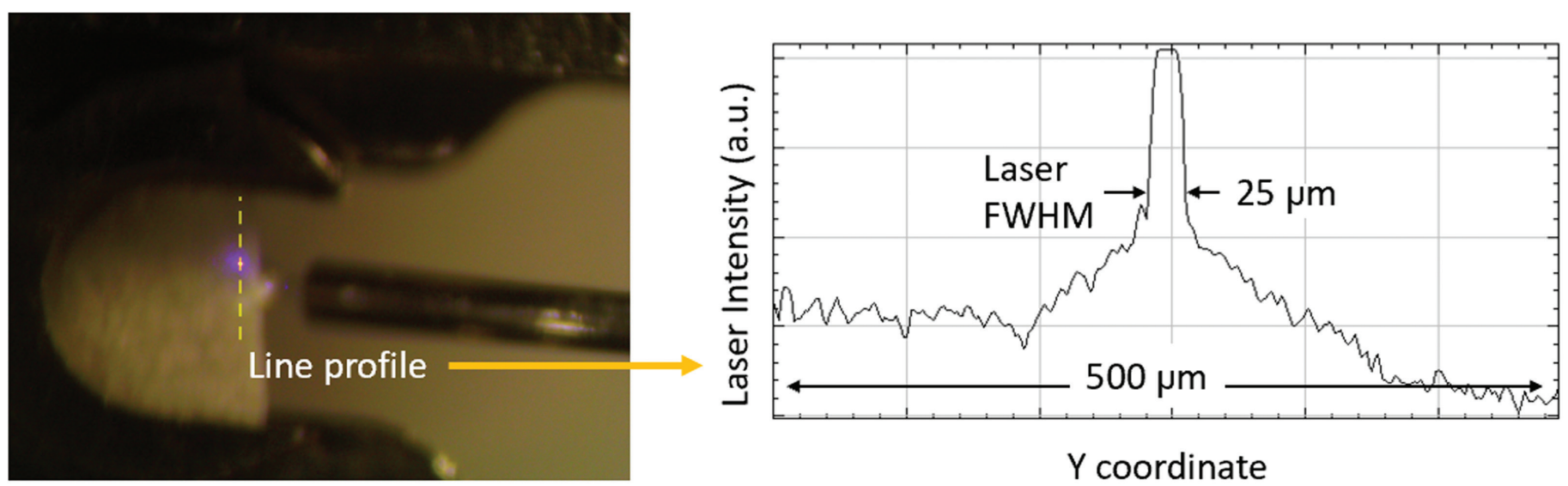

Figure 7: (left) Image of $405 \mathrm{~nm}$ laser spot on filter paper and (right) line profile of the laser spot.
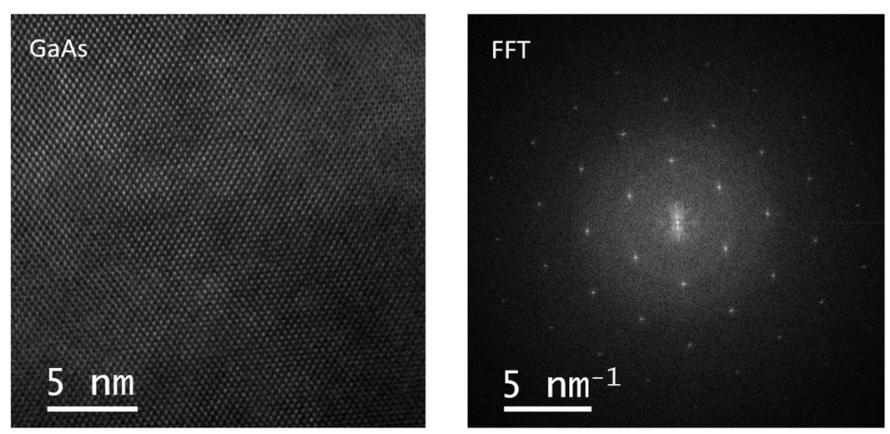

Figure 8: (left) HRTEM image of GaAs and (right) corresponding FFT.

authors would also like to thank Lakhbir Johal (Stanford Thermosciences Machine Shop) for help with machining and fabrication.

This research was funded by the Defense University Research Instrumentation Program (DURIP) under Grant No. N00014-19-1-2463, as part of the Center for Enhanced Nanofluidic Transport (CENT), an Energy Frontier Research Center funded by the U.S. Department of Energy (DOE), Office of Science, Basic Energy Sciences (BES), under Award No. DESC0019112, and using financial support by the Air Force Office of Scientific Research under grant number FA9550-19-10309. Part of this research was performed at the Stanford Nano Shared Facilities (SNSF), supported by the National Science Foundation under Award No. ECCS-1542152.

\section{References}

[1] OL Krivanek et al., Nature 514 (2014) https://doi. org/10.1038/nature13870.

[2] Z Zhang et al., Nano Lett 21 (2021) https://doi.org/10.1021/ acs.nanolett.0c03993.

[3] Z Zhang et al., Appl Phys Lett 118 (2021) htps://doi. org/10.1063/5.0029979.

[4] BK Miller and PA Crozier, Microsc Microanal 19 (2013) https://doi.org/10.1017/S1431927612014122.

[5] M Picher et al., Ultramicroscopy 150 (2015) https://doi. org/10.1016/j.ultramic.2014.11.023.

[6] D Shindo et al., J Electron Microsc 58 (2009) https://doi. org/10.1093/jmicro/dfp018.

[7] F Cavalca et al., Nanotechnol 23 (2012) https://doi. org/10.1088/0957-4484/23/7/075705.

[8] C Liu et al., Rev Sci Instrum 92 (2021) https://doi. org/10.1063/5.0031115.

[9] Thorlabs Part No. FN96A: Guide to Connectorization and Polishing Optical Fibers, https://www.thorlabs.com/ thorproduct.cfm?partnumber=FN96A.

[10] JH Hubbell and SM Seltzer, X-Ray Mass Attenuation Coefficients, NIST Standard Reference Database 126 (2004), https://www.nist.gov/pml/x-ray-mass-attenuationcoefficients.

\section{Refurbishing or servicing an electron microscope? Scope us out for your ion pump needs.}

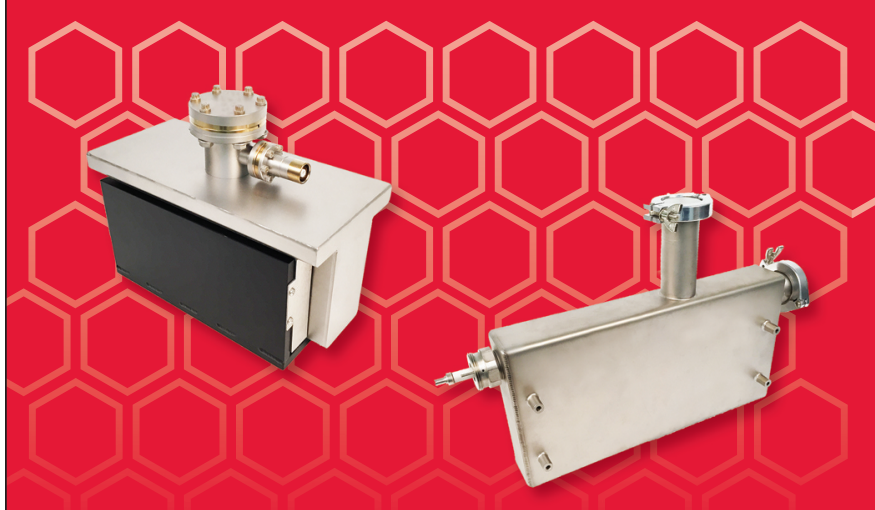

New | Rebuilt | Rebuilding Services DUOCKODNAM

800.448.8811

www - duniway - oom 


\section{Hohtrolity EBSD Aholys}



\section{Clarity EBSD Analysis System}

The world's first commercial direct detection system to produce high-fidelity EBSD patterns. This revolutionary system eliminates detector noise and distortions, opening new doors to unparalleled EBSD pattern quality and sensitivity.

- Direct electron detection of EBSD patterns

- Zero distortion for ultimate sharpness and maximum details

- No read noise for high sensitivity
- Single-electron detection

- True quantitative intensity measurements

- Ideal for beam-sensitive materials and HR-EBSD

For more information about the Clarity EBSD Analysis System, visit edax.com/clarity. 